\title{
Antiphospholipid Syndrome (APS) - An Update on Clinical Features and Treatment Options
}

\author{
Mamatha Katikaneni ${ }^{1}$, Meera Gangam ${ }^{1}$, Seth Mark Berney ${ }^{2}$ and Sarwat Umer ${ }^{*}, 1$ \\ ${ }^{I}$ Center of Excellence for Arthritis and Rheumatology, LSU Health Shreveport, Shreveport, Louisiana \\ ${ }^{2}$ Division of Rheumatology, University of Arkansas for Medical Sciences, Little Rock, Arkansas
}

\begin{abstract}
Antiphospholipid syndrome (APS) is an autoantibody disorder characterized by the presence of antiphospholipid (APL) antibodies and heterogeneous clinical manifestations. Patients may present with recurrent thrombosis, obstetric morbidity, cardiac valvular lesions, thrombocytopenia, skin lesions, renal or neurologic abnormalities. We provide a comprehensive review of these diverse clinical features except renal and obstetric complications. Treatment of APS can be challenging as one tries to balance the benefit of anticoagulation therapy in this hypercoagulable state while minimizing the risk of bleeding. We discuss the various therapeutic options including the role of aspirin, warfarin, low molecular weight heparin, new direct thrombin inhibitors, hydroxychloroquine, intravenous gamma globulin, rituximab and others. Lower risk APS patients (i.e. first venous thrombosis) should receive warfarin with a target INR of 2.0-3.0. Higher risk patients (i.e. arterial thrombosis or recurrent venous events) have a target INR of >3.0. Currently, warfarin remains the mainstay in treatment of APS. Because of lack of adequate data, the newer oral direct inhibitors should be considered only when there is a known allergy/ intolerance or poor control with warfarin. Additional vascular and thrombotic risk factors should be aggressively reduced. Further studies involving large number of APS patients, diagnosed according to accepted criteria, are needed to better define the role of newer anticoagulants and other novel therapies.
\end{abstract}

Keywords: Antiphospholipid syndrome (APS), antiphospholipid (aPL) antibodies, catastrophic antiphospholipid syndrome (CAPS).

\section{INTRODUCTION}

Antiphospholipid Syndrome (APS) is an autoantibody disorder which primarily affects the platelets, resulting in recurrent thrombosis and its sequelae such as pregnancy losses. APS was first described by Hughes, Harris and Gharavi [1]. These antibodies are directed against anionic phospholipids (PL) or protein-phospholipid complexes. It is one of the most common causes of acquired thrombophilia [1]. Preliminary classification criteria (Sapporo criteria) were developed in the 1999 international consensus meeting and updated in 2006 [2] (Table 1).

Antiphospholipid syndrome has been divided into primary and secondary APS. Primary APS is characterized by the presence of antiphospholipid antibody (aPL) and its clinical/ lab sequelae without evidence of an associated rheumatic disorder. Secondary antiphospholipid syndrome is seen with a coexisting connective tissue disease such as systemic lupus erythematosus or rheumatoid arthritis. However, the clinical and lab features are similar in both syndromes; hence this distinction was eliminated in the 2006 updated classification criteria.

*Address correspondence to this author at the Center of Excellence for Arthritis and Rheumatology, LSU Health Shreveport, 1501 Kings Highway, Shreveport, Louisiana 71103, USA; Tel: Tel: 501-686-5586;

Fax: 318-675-6980; E-mail: sumer@1suhsc.edu

\section{CLINICAL FEATURES}

The clinical features of APS occur as a consequence of large, medium and small vessel thrombosis and in some instances, due to direct antibody interaction with cells such as neurons [3, 4]. The most common manifestation, in the cohort described by Cervera and colleagues, was deep venous thrombosis (DVT), followed by thrombocytopenia, livedo reticularis, stroke, superficial thrombophlebitis, pulmonary embolism (PE), fetal loss and transient ischemic stroke (TIA) [5].

\section{Vascular Manifestations}

Deep venous thrombosis (DVT) of the lower extremities is the most common manifestation of APS observed in the Euro phospholipid project by Cervera and colleagues. This can lead to pulmonary embolism and subsequent pulmonary hypertension. Thrombosis may present at unusual locations such as superior vena cava, inferior vena cava, hepatic, portal (Budd Chiari syndrome), jugular, subclavian, renal, retinal, cerebral veins and superior sagittal vein. The risk of recurrent venous thromboembolism (VTE) is also increased with the presence of phospholipid antibodies [6,7].

Stroke and TIA are the most common manifestations of arterial thrombosis. In a young patient of less than age 35, with no other risk factors for atherosclerotic disease, these clinical events should prompt the evaluation for APS and 
Table 1. Revised classification criteria for the antiphospholipid syndrome (APS).

Antiphospholipid antibody syndrome (APS) is present if at least one of the clinical criteria and one of the laboratory criteria that follow are met ${ }^{\mathrm{a}}$ :

\section{Clinical criteria}

\section{Vascular thrombosis ${ }^{b}$}

One or more clinical episodes ${ }^{\mathrm{c}}$ of arterial, venous, or small vessel thrombosis ${ }^{\mathrm{d}}$, in any tissue or organ. Thrombosis must be confirmed by objective validated criteria (i.e. unequivocal findings of appropriate imaging studies or histopathology). For histopathological confirmation, thrombosis should be present without significant evidence of inflammation in the vessel wall.

\section{Pregnancy morbidity}

(a) One or more unexplained deaths of a morphologically normal fetus at or beyond the 10th week of gestation, with normal fetal morphology documented by ultrasound or by direct examination of the fetus, or

(b) One or more premature births of a morphologically normal neonate before the 34th week of gestation because of: (i) eclampsia or severe preeclampsia defined according to standard definitions, or (ii) recognized features of placental insufficiency ${ }^{\mathrm{e}}$, or

(c) Three or more unexplained consecutive spontaneous abortions before the 10th week of gestation, with maternal anatomic or hormonal abnormalities and paternal and maternal chromosomal causes excluded ${ }^{\mathrm{f}}$.

\section{Laboratory criteria ${ }^{\mathrm{g}}$}

1. Lupus anticoagulant (LA) present in plasma, on two or more occasions at least 12 weeks apart, detected according to the guidelines of the International Society on Thrombosis and Haemostasis (Scientific Subcommittee on LAs/phospholipid-dependent antibodies).

2. Anticardiolipin (aCL) antibody of $\operatorname{IgG}$ and/or IgM isotype in serum or plasma, present in medium or high titer (i.e. $>40$ GPL or MPL, or $>$ the 99 th percentile), on two or more occasions, at least 12 weeks apart, measured by a standardized ELISA.

3. Anti-b2 glycoprotein-I antibody of IgG and/or IgM isotype in serum or plasma (in titer $>$ the 99th percentile), present on two or more occasions, at least 12 weeks apart, measured by a standardized ELISA, according to recommended procedures.

${ }^{a}$ Classification of APS should be avoided if less than 12 weeks or more than 5 years separate the positive aPL test and the clinical manifestation.

${ }^{\mathrm{b}}$ Coexisting inherited or acquired factors for thrombosis are not reasons for excluding patients from APS trials. However, two subgroups of APS patients should be recognized, according to: (a) the presence, and (b) the absence of additional risk factors for thrombosis. Such cases include: age ( $>55$ in men, and $>65$ in women), and the presence of any of the established risk factors for cardiovascular disease (hypertension, diabetes mellitus, elevated LDL or low HDL cholesterol, cigarette smoking, family history of premature cardiovascular disease, body mass index $\geq 30 \mathrm{~kg} / \mathrm{m} 2$, microalbuminuria, estimated GFR $<60 \mathrm{~mL} / \mathrm{min} / 1.73 \mathrm{~m}^{2}$, inherited thrombophilias, oral contraceptives, nephrotic syndrome, malignancy, immobilization, and surgery. Thus, patients who fulfill criteria should be stratified according to contributing causes of thrombosis.

${ }^{\mathrm{C}} \mathrm{A}$ thrombotic episode in the past could be considered as a clinical criterion, provided that thrombosis is proved by appropriate diagnostic means and that no alternative diagnosis or cause of thrombosis is found.

${ }^{\mathrm{d}}$ Superficial venous thrombosis is not included in the clinical criteria.

${ }^{e}$ Generally accepted features of placental insufficiency include: (i) abnormal or non-reassuring fetal surveillance test(s), e.g. a non-reactive non-stress test, suggestive of fetal hypoxemia, (ii) abnormal Doppler flow velocimetry waveform analysis suggestive of fetal hypoxemia, e.g. absent end-diastolic flow in the umbilical artery, (iii) oligohydramnios, e.g. an amniotic fluid index of $5 \mathrm{~cm}$ or less, or (iv) a postnatal birth weight less than the 10th percentile for the gestational age.

${ }^{f}$ In studies of populations of patients who have more than one type of pregnancy morbidity, investigators are strongly encouraged to stratify groups of subjects according to $\mathrm{a}, \mathrm{b}$ or $\mathrm{c}$ above.

${ }^{\mathrm{g}}$ Investigators are strongly advised to classify APS patients in studies into one of the following categories: I, more than one laboratory criteria present (any combination); IIa, LA present alone; IIb, aCL antibody present alone; IIc, anti-b2glycoprotein-I antibody present alone.

other thrombophilias. Other potential sites include the brachial, subclavian, axillary, aorta, iliac, femoral, renal, mesenteric, retinal and peripheral arteries [7].

\section{Cardiac Manifestations}

Cardiac features range from asymptomatic valve lesions to accelerated atherosclerosis, myocardial infarction, intra cardiac thrombus, cardiac vegetations causing recurrent strokes, pulmonary hypertension, cardiomyopathy and diastolic dysfunction. Significant evidence exists that aPL antibodies are directly related to increased atherogenesis [810]. A study suggested that anticardiolipin (aCL) IgG titers correlate with the extent of intima media thickness (IMT) in all carotid segments [11] while another study contradicted their findings [8]. Myocardial infarction (MI) occurred in up to $5.5 \%$ of the patients at some stage and was the presenting manifestation in $2.8 \%$ [5]. Patients with APS who undergo intervention for acute MI also appear to have a higher risk of thrombosis leading to adverse cardiac outcomes [12]. This may also cause premature restenosis of the vein grafts used in coronary artery bypass surgery [13]. APS can cause valve abnormalities (35-50\%) ranging from valve thickening and regurgitations (more commonly), valve vegetations (also called the "Libman Sacks endocarditis") and stenosis (rare) $[14,15]$. These valve abnormalities can lead to an increased risk of embolism and rarely (4-6\%) can 
require replacement. The mitral valve is most commonly affected followed by the aortic valve [13].

\section{Pulmonary Manifestations}

The most common pulmonary manifestations are pulmonary thromboembolism ( $9 \%$ at disease diagnosis) and the resulting pulmonary hypertension $(2.2 \%)$. Other less common manifestations are in-situ pulmonary artery thrombosis, acute respiratory distress syndrome (ARDS), alveolar hemorrhage, capillaritis and fibrosing alveolitis. Approximately 3\% of cases can develop chronic pulmonary thromboembolic disease with resulting right heart strain and failure (CTEPH or chronic thromboembolic pulmonary hypertension). Patients with CTEPH may not have had a documented prior PE, but they should be evaluated. Emboli from tricuspid or pulmonary valvular lesions can also contribute to the development of pulmonary hypertension $[16,17]$.

Acute respiratory distress syndrome (ARDS) is usually present in the setting of catastrophic antiphospholipid syndrome (CAPS) and results from small vessel thrombosis and systemic inflammatory response (SIRS). Diffuse alveolar hemorrhage can occasionally present as the initial manifestation and cause cough, dyspnea and fever with or without hemoptysis and may progress to acute respiratory failure. Patients are hypoxic and become anemic with rapidly developing alveolar infiltrates on chest imaging. Bronchoscopy with bronchoalveolar lavage (BAL) is helpful in evaluating for other conditions and typically contains hemosiderin-laden macrophages. In ARDS related to CAPS, alveolar hemorrhage, microvascular thrombosis with or without capillaritis may be seen histologically [17, 18]. A postpartum syndrome is also reported in the literature in which patients with aPL antibodies present with spiking fevers, pleuritic chest pain and dyspnea and have chest radiographs with pleural effusions and patchy infiltrates.

\section{Dermatological Manifestations}

Cutaneous features occurred in $49 \%$ of the patients and represent the initial APS manifestation in approximately $30.5 \%$ [5]. Livedo reticularis (LR) is the most common dermatological manifestation, present in $18-26 \%$ of patients with APS [19]. The International Consensus statement on an update of the classification criteria for definite APS, defined livedo reticularis as violaceous, red or blue, reticular or mottled pattern of the skin of trunk, arms or legs and persistent, not reversible with rewarming. Pathologic changes include partial or complete occlusion of the lumen of small to medium sized arteries and/or arterioles at the dermis-subcutis border with no evidence of perivascular inflammatory infiltrate. Direct immunofluorescence examination is negative and is usually not needed for diagnosis. Livedo reticularis is not specific for the antiphospholipid syndrome and can be seen in other conditions such as vasculitis, drug reactions and as a normal physiological response to cold exposure. The LR associated with vasculitis is more infiltrated; that associated with cholesterol emboli is painful and physiological LR occurs more on the exposed areas and is not found on the trunk and buttocks [20]. Livedo reticularis is slightly more common in secondary APS due to SLE. An increased association of cardiolipin IgG with LR has been reported [19]. The LR lesions can lead to ischemia and tissue infarction, called livedo vasculitis which can cause purpuric macules, cutaneous nodules, and/or painful ulcerations. LR may also indicate patients at an increased risk of cerebral and ocular ischemic events, arterial thrombosis, systemic hypertension, heart valve abnormalities, Raynaud's and pregnancy morbidity [19]. Sneddon syndrome is a rare and potentially severe condition characterized by livedo reticularis and multiple cerebrovascular accidents (CVA) which is occasionally associated with phospholipid antibodies.

Non-livedo reticularis skin ulcers were reported in 5.5\% [5] and can be the presenting manifestation in about 3.9\%. They result from either circumscribed skin necrosis or post phlebitis ulcers. The skin necrosis ulcers usually present in the lower extremities around the ankles, feet and calves and are usually preceded by necrotizing purpura, are painful and heal leaving an atrophie blanche like scar [19]. Post phlebitis ulcers can occur in the lower extremities after many years of recurrent attacks of phlebitis. APS should be in the differential for Pyoderma Gangrenosum like lesions unresponsive to the usual treatment [21]. Subungal splinter hemorrhages may be seen along with thrombotic events elsewhere [19]. Thrombocytopenic purpura may be observed with low platelet counts and more commonly associated with secondary APS associated with SLE.

\section{Neurologic Manifestations}

Stroke and TIA occurred in about $19.8 \%$ and $11.1 \%$ respectively of the patients in the Euro- phospholipid cohort and was the most common cause of recurrent thrombosis [22]. It commonly affects the middle cerebral artery (MCA) territory but can involve any area [23]. The symptoms can include amarausis fugax, transient paresthesias, weakness, vertigo or transient global ischemia. Recurrent episodes can lead to multi infarct dementia and cognitive dysfunction. Intractable headaches, cognitive dysfunction resulting in poor memory, difficulty in concentration have been described [24]. Practitioners should have a low threshold for checking for phospholipid antibodies in a young patient who presents with stroke without obvious risk factors. Emboli from the heart especially in the presence of valve lesions can be the other source for CVA. Patients may rarely present with sagittal venous sinus thrombosis and acute ischemic encephalopathy [25]. In addition to thrombosis, direct immune interaction of the aPL antibodies with the neurons, is also thought to contribute to manifestations such as seizures [3], chorea [4] and transverse myelitis. The transverse myelitis occurring in APS is typically monophasic but rarely recurrent corticosteroid responsive longitudinally extensive transverse myelitis has also been observed [26]. Many cases of antiphospholipid syndrome are initially diagnosed as remitting-relapsing multiple sclerosis (MS). The magnetic resonance imaging (MRI) from MS is frequently indistinguishable from that due to APS. However, a careful history, the presence of aPL antibodies and the response to anticoagulation therapy may help in differentiating the two [27]. Idiopathic intracranial hypertension, sudden onset sensorineural hearing loss due to impaired circulation in the inner ear, transient blurred vision, 
transient diplopia, Guillain Barre-like syndrome, transient global amnesia, neuropathy, dystonia and a Parkinson-like syndrome have been described. The presence of aPL antibodies in SLE was associated with higher incidence of neurological complications [28]. MRI of the brain may show lesions consistent with the infarct or reveal small foci of high signal in the white matter consistent with small vessel disease [27].

\section{Hematologic Manifestations}

A consumptive thrombocytopenia with antiphospholipid antibodies is common and is typically mild. The platelet count ranges between $100,000-150,000 / \mathrm{microL}$ and is usually not severe enough to cause bleeding. Coombs positive hemolytic anemia has also been described. Phospholipid antibodies and hypercoagulability are also implicated in the development of osteonecrosis, and rarely with bone marrow necrosis (necrosis of the bone marrow with the preservation of bone) [29].

\section{Endocrine Manifestations}

Primary adrenal insufficiency or adrenal failure is the most common endocrine manifestation and can sometimes be the presenting feature of APS (4\%). Imaging reveals adrenal hemorrhage, which is thought to be due to thrombosis followed by secondary hemorrhage [30]. It is also frequently present as a part of catastrophic antiphospholipid syndrome. Cases of ovarian vein thrombosis [31], testicular vein thrombosis [32] and pituitary thrombosis causing hypopituitarism have been reported [33, 34].

\section{Catastrophic Antiphospholipid Syndrome (CAPS)}

Catastrophic antiphospholipid syndrome (CAPS) is a rare (less than 1\%) manifestation with a very high mortality. CAPS causes thrombosis in three or more organs within one week with the histological demonstration of thrombi in the small vessels of the involved organs and presence of aPL antibodies in high titers. It is classified as probable or definite CAPS (Table 2).
It differs from other manifestations of APS by the presence of small vessel involvement and micro thrombi in the involved organs and associated with the systemic inflammatory response (SIRS). Patients are very sick and usually in the intensive care units. Infections, sudden withdrawal of anticoagulation, oral contraceptives, surgical procedures, and obstetric complications have been implicated in triggering these episodes [35]. The clinical manifestations depend on the organs involved. Encephalopathy, cardiac dysfunction and acute respiratory distress syndrome (ARDS) occur due to the cytokine cascade released from the necrotic organs. The kidneys, lungs, bowel, heart, skin and brain are the most commonly affected organs. Other organs involved are adrenal, testis, spleen, pancreas and skin. An analysis of mortality and prognostic factors in 250 patients with CAPS indicated that the presence of SLE was related to a higher mortality rate. Cerebral involvement (primarily stroke) was considered the single main cause of death, present in $27.1 \%$ of patients. However, cardiac and pulmonary conditions together represented the main cause of death, present in $29.6 \%$ of patients. Up to $50 \%$ of patients with CAPS have thrombocytopenia, one third develop hemolysis, and some may have features of disseminated intravascular coagulation [36]. The CAPS registry project group was created in 2001 and periodic analysis of this data helps us to better understand the disease and treatment [37].

\section{TREATMENT}

The clinical presentation and the severity of the patient's antiphospholipid syndrome (APS) determine the therapy. The main principle of APS management focuses on attenuating the hypercoagulable state while balancing the risks of anticoagulation therapy. Treatment of APS can be challenging due to recurrent thrombosis despite seemingly appropriate anticoagulation, and may require the coadministration of immunomodulators or antiplatelet agents. Recent progress in understanding the pathogenic mechanisms of APS has expanded the horizon for targeted therapies such as anti-inflammatory and immunosuppressive medications. The therapeutic options will be discussed here followed by the current recommendations for treatment based on each organ involvement.

Table 2. Preliminary criteria for diagnosis of catastrophic APS [38].

1. Evidence of involvement of three or more organs, systems and/or tissues

2. Development of manifestations simultaneously or in less than a week

3. Confirmation by histopathology of small vessel occlusion in at least one organ or tissue

4. Laboratory confirmation of the presence of antiphospholipid antibodies (lupus anticoagulant and/or anticardiolipin antibodies)

\section{Definite catastrophic APS}

- All 4 criteria

Probable catastrophic APS

- All 4 criteria, except for only two organs, systems and/or tissues involvement

- All 4 criteria, except for the absence of laboratory confirmation at least 6 weeks apart due to the early death of a patient never previously tested for aPL prior to the catastrophic APS event

- $\quad$ Criteria 1,2 and 4

- Criteria 1,3 and 4 and the development of a third event in more than a week but less than a month, despite anticoagulation 


\section{THERAPEUTIC OPTIONS}

\section{Acetylsalicylic Acid (Aspirin)}

Aspirin acts as an antiplatelet agent by inhibiting thromboxane production thus preventing platelets from binding to each other. In the Antiphospholipid Antibody Acetylsalicylic Acid (APLASA) trial, aspirin $81 \mathrm{mg}$ daily (considered low dose) did not show a benefit in the primary prophylaxis of APS [40]. In contrast, a meta-analysis of 11 observational and interventional studies suggested that the risk of first thrombotic event is significantly decreased by low dose aspirin among asymptomatic aPL individuals, patients with SLE or obstetric APS [39]. However, aspirin is recommended for all adults by the United States Preventive Services Task Force (USPSTF) for the prophylaxis of coronary artery disease due to its beneficial antiplatelet effect and minimal complications and cost. Hence, we usually recommend aspirin at a dose $81 \mathrm{mg}$ daily for all patients with APL unless the patient has a contraindication to its use. The major side effects reported with high doses of aspirin are gastrointestinal ulcers, bleeding, renal dysfunction, asthma exacerbation in aspirin sensitive patients and tinnitus.

\section{Anticoagulation}

Currently, the mainstay of treatment for acute thrombotic APS is heparin followed by long term anticoagulation with vitamin $\mathrm{K}$ antagonists such as warfarin.

\section{Unfractionated and Low Molecular Weight (LMW) Heparin}

Heparin is an injectable anticoagulant that binds to Antithrombin III and inactivates Thrombin. It also binds with other coagulations factors, predominantly Factor Xa and inhibits the conversion of fibrinogen to fibrin to prevent clot formation. The unfractionated heparin is used as a continuous intravenous infusion due to its short half-life and hence is a bridging therapy till therapeutic anticoagulation with an oral anticoagulant such as warfarin is achieved. The infusion amount is titrated to achieve an aPTT (activated partial thromboplastin time) double to the baseline aPTT [42]. The LMW heparins include enoxaparin $(1 \mathrm{mg} / \mathrm{kg}$ subcutaneous every 12 hours) and dalteparin (200 units $/ \mathrm{kg} /$ day subcutaneous). The adverse reactions of unfractionated and LMW heparins include bleeding, heparin induced thrombocytopenia, hypersensitivity reactions (rarely) and osteoporosis (with prolonged use). The cost of Enoxaparin is $\$ 10$ and Dalteparin is $\$ 78$ for each prefilled syringe.

\section{Warfarin}

Warfarin depletes functional vitamin $\mathrm{K}$ reserves, inhibiting production of the coagulation factors II, VII, IX, X and the anticoagulation factors Protein $\mathrm{C}$ and Protein $\mathrm{S}$ by the liver. The current recommendation is to maintain an international normalized ratio (INR) of 2.5 (2.0-3.0) for an indefinite period of time following the first episode of venous thromboembolism (VTE) or recurrent VTE events while off anticoagulation. With arterial thrombosis, the optimal intensity of anticoagulation is not established due to lack of sufficient number of patients in clinical trials however experts believe that INR should be maintained $>3.0$ [43]. Warfarin has many concerns including a narrow therapeutic window, significant risk of bleeding complications, frequent laboratory monitoring of INR, slow onset and offset of action and numerous drug and dietary interactions. The adverse reactions that have been reported include bleeding, skin necrosis, hypersensitivity, elevated transaminases and cholestatic hepatitis. The use of warfarin is contra-indicated in any patients with pregnancy, hypersensitivity, life-threatening bleeding tendencies, frequent falls and non-compliance.

\section{Newer Anticoagulants}

The newer oral anticoagulants include the direct thrombin inhibitor dabigatran etexilate (Pradaxa) and direct anti-Xa inhibitors rivaroxaban (Xeralto), apixaban (Eliquis) and edoxaban (Lixiana). The major advantage of these newer agents is the fewer reported drug and dietary interactions and the lack of monitoring due to a more predictable anticoagulant effect. The EINSTEIN-PE and EINSTEINDVT trials are international multicenter randomized controlled trials that evaluated Rivaroxaban in the treatment and prevention of DVT and acute symptomatic PE. Large phase III clinical trials [45-47] demonstrated the efficacy of dabigatran and rivaroxaban and their low incidences of major bleeding compared to Warfarin in adults. However, these trials may not be directly applicable to patients with APS. The Rivaroxaban in Antiphospholipid Syndrome Trial (RAPS) is a prospective non-inferiority randomized controlled trial of warfarin versus rivaroxaban in patients with venous thrombotic APS, with or without SLE being maintained at target INR of 2.5 is currently in progress. Hence, the 14th International Congress on Antiphospholipid Antibodies Task Force (ICAATF) recommended that warfarin remains the mainstay in treatment of APS and newer oral direct inhibitors should be considered only when there is a known allergy/ intolerance or poor control with warfarin due to lack of adequate data [44]. Bleeding is a risk with any form of anticoagulation. The risk of major bleeding complications with newer oral anticoagulants at therapeutic dose are slightly lower compared to warfarin in the phase III clinical trials like ROCKET-AF (rivaroxaban), RE-COVER (dabigatran), RE-LY AF (dabigatran) and ARISTOTLE (apixaban) trials [48].

Dosing and approximate cost for 1 month supply:

Dabigatran $150 \mathrm{mg}$ twice daily, $\$ 596$

Rivaroxaban $20 \mathrm{mg}$ once daily, $\$ 313$

Apixaban $5 \mathrm{mg}$ twice daily, $\$ 313$

Edoxaban $60 \mathrm{mg}$ once daily, $\$ 1,330$.

\section{Hydroxychloroquine (HCQ)}

Wallace et al. first reported a protective effect of HCQ against thrombosis, which was then confirmed by several other prospective and retrospective studies [49, 50]. However, it is unclear if the benefit seen by HCQ against thrombosis in SLE is related to its effect on aPL, lupus or other cardiovascular risk factors because HCQ also appears to lower lipid and glucose levels. The proposed antithrombotic mechanisms of HCQ include inhibition of 
platelet aggregation and adhesion, cholesterol lowering, blockade of aPL production and binding of aPL-B2GPI complexes to phospholipid surfaces [44]. A multicenter, international prospective randomized controlled trial of HCQ for primary thrombosis prevention in persistently aPL positive patients without other systemic autoimmune diseases (HCQ Trial) is in progress. The $14^{\text {th }}$ ICAATF recommends HCQ in all aPL positive SLE patients, however, no compelling data exists regarding prescribing HCQ in patients without an associated autoimmune disease. Because of its good safety profile, clinicians should consider HCQ as adjunctive therapy in refractory APS. The use of HCQ for more than 10 years increases the risk of skin, retinal and muscle toxicity [44]. The dosage of HCQ most commonly used is $200 \mathrm{mg}$ twice a day.

\section{3-Hydroxy-3-Methyl-Glutaryl-CoA (HMG Co Reductase Inhibitors - (Statins)}

Statins have been shown to decrease aPL-induced endothelial cell activation via inhibition of the expression of adhesion molecules and IL-6 and by reversal of Tissue Factor upregulation. They also decrease oxidative stress and inflammation, inhibit the thrombogenic response and have other beneficial immune system effects [51-53]. In APS, statins may interfere with monocyte, lymphocyte and endothelial cell function, thus helping to prevent thrombosis $[53,54]$. Preliminary results of an on-going phase II trial investigating the levels of prothrombotic/ proinflammatory biomarkers in aPL patients, showed a significant decrease in vascular endothelial growth factor, soluble tissue factors and tumor necrosis factor with fluvastatin. However, randomized controlled trials are necessary to support the utility of statins in APS. The $14^{\text {th }}$ ICAATF does not recommend statins use in APS patients without hyperlipidemia despite the beneficial effect on the inflammation and thrombotic pathways because no trials exist which translate this benefit to clinically significant improvement [44]. However, refractory APS patients may derive benefit from the addition of statins. The major side effects of statins include myopathy and elevated transaminases.

\section{Rituximab (RTX)}

Rituximab is a monoclonal antibody directed against the CD20 receptor on $\mathrm{B}$ lymphocytes resulting in complement dependent $\mathrm{B}$ cell cytotoxicity. Its precise mechanism of action in APS is unknown. However, it may include decreasing the production and amount of circulating APL antibodies or decreasing pro-inflammatory cytokines. Rituximab has been used in patients with severe cases of APS with thrombocytopenia or autoimmune hemolytic anemia resistant to standard therapy [55-57]. A pilot open label phase II trial (RITAPS) of RTX (1000 mg on Day 1 and 15) for non-criteria manifestations of APS without any systemic autoimmune disease suggested it was safe. It showed a $50 \%$ response of thrombocytopenia, skin ulcers, partial response to nephropathy, cognitive dysfunction. However, the major limitations of the RITAPS trial are small sample size, short follow up duration (12 months) [58]. The $14^{\text {th }}$ ICAATF recommends reserving the use of Rituximab for refractory APS in patients with hematologic and microangiopathic manifestations [44]. The major side effects reported include infusion reactions, infections and cytopenias. The cost of one cycle (2 doses) is approximately $\$ 14,900$.

\section{Complement Inhibition (Eculizumab)}

Eculizumab is a recombinant humanized monoclonal antibody that binds to the terminal complement protein $\mathrm{C} 5$ and inhibits its cleavage. Increasing evidence linking the coagulation cascade and complement activation has caused complement inhibition to receive a lot of attention recently. Many studies on mouse models suggested that complement proteins are important mediators in fetal damage in APS. Heparin appears to inhibit complement activation, and mice deficient in complement were resistant to thrombosis in aPL. However, no studies in humans exist except case reports detailing Eculizumab use in CAPS [45, 59, 60]. Hence, because data is needed to show definite benefit, the current recommendation reserves complement inhibition as adjuvant therapy in patients with refractory APS [44]. No data exists regarding the dosing. The major adverse event is infections with encapsulated organisms. Each dose costs up to $\$ 20,000$.

\section{Vitamin D}

In vitro APS studies showed that Vitamin D may function as an anti-thrombotic immunomodulator and low vitamin D levels correlated with thrombosis [61]. The 14th ICAATF suggested replacement/ correction of vitamin D levels in patients with APS and low vitamin D levels using the general population guidelines [44].

\section{Intravenous Immunoglobulins (IVIG):}

The mechanism of action of IVIG remains unclear but appears to suppress inflammatory and immune mediated processes. A few studies showed the efficacy of IVIG as an adjuvant to conventional therapy in primary and secondary APS [62, 63], but the major limitations with use of IVIG includes the expense (approximately $\$ 15,000$ per dose) and the lack of significant evidence of efficacy. This treatment option may be reserved for patients who are refractory to anticoagulation or when additional indications such as autoimmune thrombocytopenia are present. The major side effects include anaphylaxis, renal toxicity, hemolysis, aseptic meningitis. The recommended dose of IVIG in Catastrophic APS (CAPS) is $0.4 \mathrm{~g} / \mathrm{day} / \mathrm{kg}$ body weight for $4-5$ days [64].

\section{Therapeutic Plasma Exchange (TPE)/ Plasmapheresis}

The mechanism of action and its benefit in APS is unknown but it is thought to remove cytokines and complement components. Although plasma exchange for CAPS has never been investigated in a prospective, randomized study, a review of the first 250 patients entered into the CAPS Registry noted that the combination of TPE, anticoagulants, and steroids resulted in an overall 78 percent survival [65], leading the authors to conclude that this treatment combination should be the first line of therapy for patients with CAPS. Although the optimum number of plasma exchange treatments has not been determined, the 
cost of TPE in various studies ranged from $\$ 3,000$ to $\$ 10,000$ per treatment.

\section{Others:}

Reports of tissue factor inhibition, NF-kB and P38 Mitogen activated kinase inhibitors, blocking of aPL receptors on target cells and stem cell transplantation may be future targets for therapy, but long term follow up data are required before these are considered as real treatment options [45].

\section{TREATMENT BASED ON THE CLINICAL MANI- FESTATIONS}

\section{Asymptomatic Patients with aPL - Primary Thrombo- prophylaxis}

Observational studies have shown that primary thromboprophylaxis with aspirin showed no difference in the risk of thromboembolic events. However, patients in highrisk situations (surgery, immobilization, pregnancy) experienced a $31 \%$ reduction in risk of thrombotic events $[66,67]$. The APLASA clinical trial indicated no clear advantage between placebo and aspirin $81 \mathrm{mg}$ for primary thromboprophylaxis [40]. In contrast, a meta-analysis of 11 observational and interventional studies suggested that the risk of first thrombotic event is significantly decreased by low dose aspirin among asymptomatic aPL individuals, patients with SLE or obstetric APS [39]. Hydroxychloroquine appears to prevent post-operative thrombosis after hip replacement [68] and is beneficial to patients with SLE [69]. Erkan et al. observed a decreased risk of thrombosis in asymptomatic aPL positive individuals with HCQ although $78 \%$ of patients in the study had SLE [70]. In patients with aPL and SLE, aspirin and hydroxychloroquine have shown to be effective in decreasing the overall risk of thrombosis.

\section{Vascular Thrombosis (Arterial or Venous)}

The initial treatment consists of unfractionated or low molecular weight Heparin for at least 5 days overlapping with warfarin therapy with a target INR of 2-3. The choice of therapy, intensity of anticoagulation, duration of therapy should be individualized on the basis of type of thrombosis. The recommendation for first venous thrombosis is anticoagulation with a target INR of 2-3. However, if the thrombosis was unprovoked or the patient has a high risk profile as defined as triple APL antibody positive, persistent APL antibody positive, lupus anticoagulant positive, or high titer IgG of anti cardiolipin (aCL) or anti B2Glycoprotein (B2GPI) antibodies, lifelong anticoagulation is recommended. If a known transient precipitating factor existed which has abated and/or a low risk profile is identified (defined as isolated intermittently positive aCL or Anti-B2GPI at low titers), only 3-6 months of anticoagulation with close monitoring for future events may be sufficient [41]. The recommendation for arterial thrombosis or recurrent venous thrombosis with definite APS is high intensity anticoagulation (INR $>3$ ) or the combination of an antiplatelet agent with low intensity (INR 2-3) anticoagulation. A meta-analysis done by Ruiz-Irastorza et al. showed that recurrences of arterial and venous thrombosis occurred infrequently among patients effectively receiving oral anticoagulation with a consistent INR of 3-4. Furthermore, arterial thrombosis recurrence rate was higher than venous thrombosis [71]. Many small studies report contradictory results for high intensity anticoagulation and the combination of warfarin and aspirin. However, RuizIrastorza et al's current recommendations include managing with warfarin at INR $>3$ or combination of warfarin with INR 2-3 and Aspirin $100 \mathrm{mg}$ daily [71, 72]. No data exists regarding the addition of aspirin for patients with recurrent thrombotic events while on anticoagulation achieving INR 34.

\section{Recurrent Thrombosis}

Recurrence of thrombosis must be clearly documented to assess if the symptoms are due to a new event and not just the sequelae of a previous event such as post-thrombotic syndrome (PTS) following a deep vein thrombosis (DVT). PTS occurs in the first 2 years following DVT and is seen in patients with obesity, recurrent ipsilateral DVT, sub therapeutic INR, proximal DVT and may cause leg heaviness, pain, swelling, edema, cramps, venous ectasia, ulcer or signs of lipodermatosclerosis similar to symptoms of DVT. Hence, it is essential to differentiate the two because the treatment is very different. The most effective way to prevent PTS is by using elastic compression stockings [41]. Once a recurrence of thrombosis is documented, there is a need to assess the bleeding risk and either increase anticoagulation intensity or add another agent such as aspirin, heparin, newer oral anticoagulants, triple therapy (aspirin, warfarin and clopidogrel), hydroxychloroquine, rituximab etc. The evidence suggests that the mortality due to bleeding with high intensity anticoagulation in recurrent thrombosis is much lower compared to the risk due to thrombosis. Plasma exchange and IVIG may be second line agents for refractory APS [41].

\section{Cardiac Manifestations}

Coronary Artery Disease (CAD): The recommendation is to screen and treat for all traditional risk factors including hypertension, hypercholesterolemia, smoking and diabetes. Statins are known to cause regression of atherosclerotic lesions and reduce cardiovascular complications. Hydroxychloroquine appears to provide significant cardiovascular benefit in lupus patients. Many studies suggest the beneficial role of aspirin in preventing CAD in patients with or without lupus. The committee consensus report recommended statins, folic acid, B vitamins and hydroxychloroquine for patients with an elevated cardiovascular risk [73]. In myocardial infarction patients with a high-risk profile, longterm anticoagulation with INR 3-4 or INR 2-3 plus low dose aspirin is recommended. However, if the patient undergoes a coronary intervention, they should receive a full antithrombotic regimen with warfarin, clopidogrel and aspirin [41].

Valve abnormalities: Espnola-Zavaleta et al. showed that oral antiplatelet or anticoagulation therapy does not diminish the non-infective valve lesions seen in primary APS. However, due to the risk of systemic embolization from vegetations and valve thickening, anticoagulation with 
heparin or warfarin is recommended. However, no data exists regarding anti-inflammatory or immunosuppressive therapy [13]. Furthermore, surgical excision of the vegetations may not prevent recurrence.

The "Task Force on Catastrophic Antiphospholipid Syndrome (CAPS) and Non-criteria APS Manifestations" developed the following recommendations [74]:

1. Transthoracic Echocardiogram (TTE) in all patients with a history of APS and previous arterial thrombosis.

2. Follow up serial echocardiograms in patients with abnormal heart valves to determine the need for surgery because the valve lesions can worsen despite anticoagulation.

3. The valvular lesions may predispose patients to bacterial endocarditis. Thus, prophylactic antibiotics should be used for dental and surgical procedures with increased risk for transient bacteremia.

Post-operative mortality and morbidity after valve replacement is very high due to bleeding and thrombosis, thus requiring careful monitoring of anticoagulation [75].

Intra-cardiac thrombosis: Patients with APS require screening for intra-cardiac thrombosis and treatment with intensive warfarin therapy and consult cardio-thoracic surgeon when appropriate [73].

\section{Pulmonary Manifestations}

1. Acute Pulmonary embolus (PE): Heparin acutely followed by long term warfarin for prophylaxis of recurrent $\mathrm{PE}$ with the target INR still being controversial.

2. Pulmonary hypertension due to chronic PE: Apart from chronic anticoagulation, other treatments include calcium channel blockers, bosentan, cyclophosphamide, prostacyclin or epoprostenol infusions [13]. The role of IVC filters and thromboendarterectomy is not well established. Surgical procedures such as atrial septectomy or lung transplantation have been performed in patients refractory to medical management. The mortality is very high and the outcome of pulmonary hypertension in aPL is usually fatal.

3. Pulmonary arterial thrombosis can be treated with thrombo-endarterectomy and long term anticoagulation.

4. Adult Respiratory Distress Syndrome (ARDS) has been treated with high dose IV steroids, heparin, plasmapheresis and IV cyclophosphamide but the mortality is high.

5. Intra-alveolar pulmonary hemorrhage can be treated with pulse dose steroids, IVIG, IV Cyclophosphamide and withholding anticoagulation which needs to be restarted as soon as the pulmonary status improves [17].

\section{Dermatologic Manifestations}

No treatment has consistently been effective in livedo reticularis. Patients who have Sneddon's syndrome have been shown to benefit from chronic anticoagulation. Patients should stop smoking and avoid estrogen containing contraceptives due to the high risk of arterial thrombosis. Low dose aspirin, statins, clopidogrel, and angiotensin converting enzyme inhibitors benefit is still unproven [76]. There is no consensus on the treatment of livedoid vasculopathy and only anecdotal evidence exists using antiplatelet, anticoagulant and fibrinolytic therapies. Patients with digital gangrene and widespread cutaneous necrosis should receive full anticoagulation with heparin. Plasma exchange and intravenous steroids can be added if the necrosis worsens despite anticoagulation. Illoprost can be added to these therapies to increase the healing process followed by long term treatment with warfarin. Minor lesions can be treated with combination of aspirin and dipyridamole [21].

\section{Neurologic Manifestations}

The cornerstones of treatment of neurologic manifestations are aspirin, heparin and warfarin. In some cases, clopidogrel can replace aspirin. Khamashta et al. analyzed APS patients over a period of 10 years and showed that there was a significant benefit from high intensity anticoagulation; the danger of thrombosis and stroke outweighs the risk of anticoagulant induced bleeding [77]. Patients with aPL and cerebral ischemia require lifelong high intensity anticoagulation. However, if the patient has a low risk profile without any systemic autoimmune disease, the first cardio-embolic cerebral arterial event in the presence of a reversible initiating factor, antiplatelet agent (aspirin 325 $\mathrm{mg}$ ) may be tried instead of oral anticoagulation based on the APASS study [78]. For other neuropsychiatric manifestations such as headaches and seizures, anticoagulation may be helpful in patients with severe manifestations who are unresponsive to conventional treatment [79].

\section{Hematologic Manifestations}

Patients with thrombocytopenia remain at an increased risk of thrombosis. In fact, platelet counts below 50,000 confers a significantly increased risk for bleeding. APS patients with profound thrombocytopenia should receive corticosteroids (and, if necessary, IVIG) to achieve a platelet count of greater than 50,000 . Once the platelet count is stable above 50,000, the cautious introduction of warfarin can be considered if the patient has a history of thrombosis [80]. Splenectomy in patients with refractory and severe thrombocytopenia results in a long term improvement, but these patients may be prone for arterial and portal vein thrombosis during and after splenectomy [81]. Management of hemolytic anemia in APS has been reported with corticosteroids, azathioprine, rituximab and splenectomy to be effective. Steroids and therapeutic plasma exchange is the first line treatment recommended for all patients with thrombotic microangiopathic hemolytic anemia associated with aPL [82]. 


\section{Endocrine Manifestations}

Adrenal hemorrhage/ infarction have been successfully treated with steroid replacement therapy with or without anticoagulation and aspirin. For adrenal hemorrhage associated with CAPS, IVIG and therapeutic plasma exchange (TPE) have been tried [83].

\section{Catastrophic Antiphospholipid Syndrome (CAPS)}

The goals of therapy in CAPS are to treat any precipitating factors, treat any on-going thrombotic events and suppress the "cytokine storm". The common precipitating factors for CAPS include infections (respiratory, skin, urinary tract, and sepsis), surgical procedures, malignancy, lupus flares, sudden anticoagulation withdrawal, oral contraceptives and obstetric complications. However, $35 \%$ of patients do not have any identifiable precipitating factor [38]. The evidence supporting the therapeutic options is very limited due to lack of prospective trials.

To prevent CAPS in aPL [84]:

1. Any infection, however trivial, should be treated with appropriate antibiotics.

2. Minimize surgical procedures in patients with APS.

3. Remember that thrombocytopenia doesn't protect against thrombosis.

4. Minimize stasis in the distal vascular bed and avoid tourniquets.

5. Continue physiologic and pharmacologic anticoagulation measures before and after surgery till patient becomes completely ambulatory post operatively.
6. APS patients undergoing surgery should receive parenteral anticoagulation (heparin) instead of warfarin.

7. Continuation of anticoagulation for at least 6 weeks postpartum with heparin.

8. Be aware that APS patients can develop thrombosis despite adequate anticoagulation and prophylaxis.

According to the CAPS registry, anticoagulation with corticosteroids, with or without plasma exchange/ IVIG was the most commonly used therapeutic regimen that significantly reduced patient mortality. The regimen consisted of IV heparin for 7-10 days followed by warfarin with INR goal of 3 and pulse steroids (1000 mg/day) followed by high dose IV solumedrol (1-2 mg/kg/day. The recommended dose of IVIG is $0.4 \mathrm{~g} / \mathrm{day} / \mathrm{kg}$ body weight for 5 days. In most case reports and retrospective studies, plasma exchange afforded some benefit in patients who had schistocytes. Patients with SLE and CAPS may also benefit from cyclophosphamide, rituximab, prostacyclin, ancrod, defibrotide, fibrinolytics and splenectomy [38].

Other non-specific therapies include low tidal volume ventilation, preventative measures for ventilator associated pneumonia, stress ulcer prophylaxis, hemodialysis if there is renal failure and inotropic support if there is circulatory failure [63].

CAPS recurrence is unusual and patients generally have a stable course with lifelong high intensity warfarin therapy (Fig. 1).

\section{Duration of therapy}

No evidence exists delineating a time interval beyond which the risks of anticoagulation outweigh the benefits. The

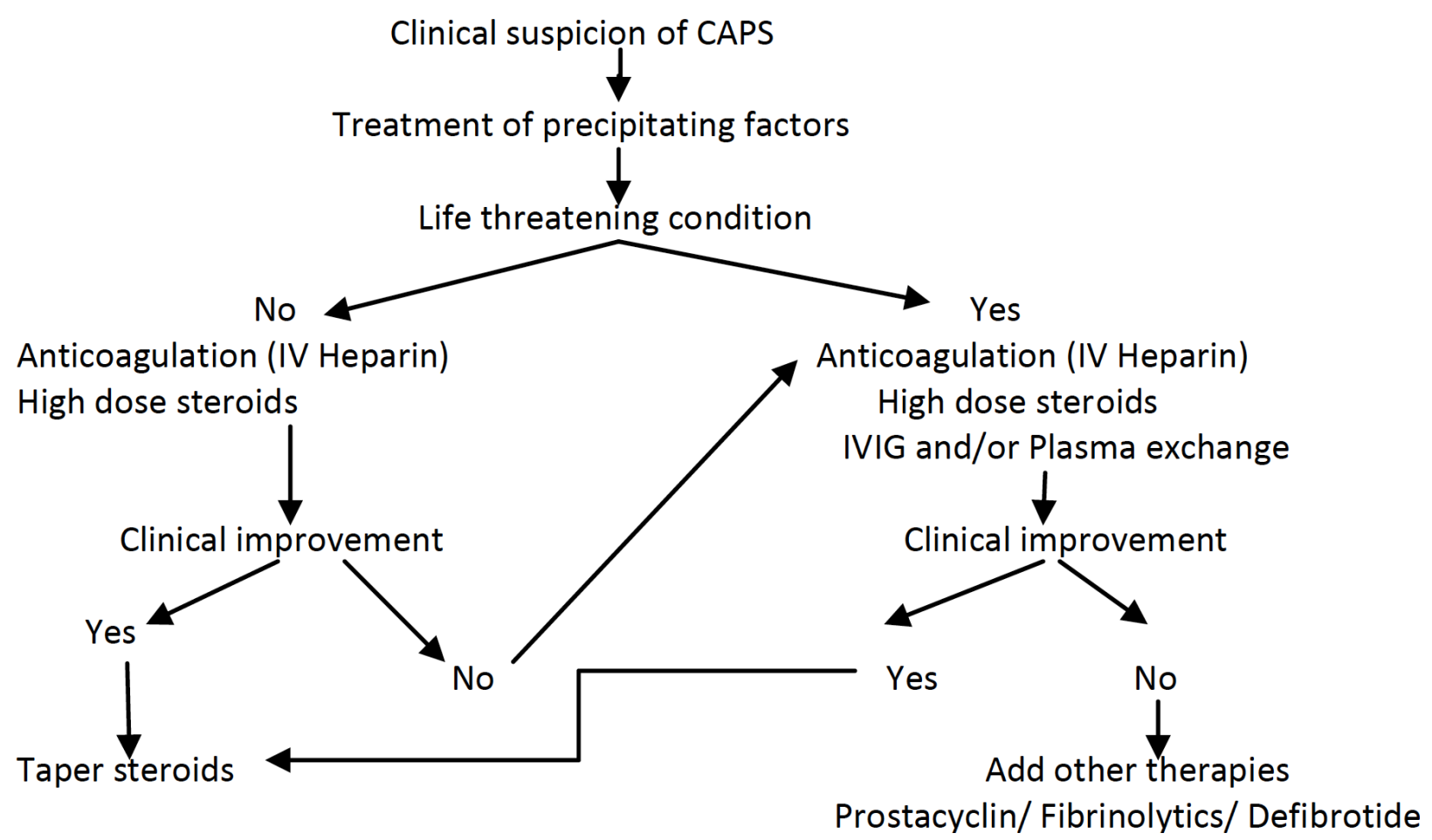

Fig. (1). Treatment algorithm for CAPS (Catastrophic APS - RA Asherson et al.) [38]. 
current recommendation of ICAATF is indefinite anticoagulation if the risk of bleeding is low and monitoring of anticoagulation is available [44]. The general consensus is lifelong antithrombotic therapy for patients with thrombotic APS and no precipitating factors. The risk of recurrent thrombosis appears to be significant 6 months after withdrawal of anticoagulation. Re-thrombosis after stopping the treatment ranged from $50 \%$ in retrospective studies to 3 $25 \%$ in prospective studies. The risk of re-thrombosis did not rise with transiently positive aCL but with persistently elevated aCL [41]. Many studies have shown that arterial thrombosis and triple positive aPL have a high risk for recurrence [85]. The ICAATF recommends that patients with their first venous thrombus and a known precipitating factor along with a low risk aPL profile, 3-6 months of anticoagulant therapy may be sufficient [44]. However, the question of whether treatment can be stopped after aPL becomes negative remains unanswered and there is a need for randomized controlled trials to determine the safety of this approach.

\section{CONFLICT OF INTEREST}

The authors confirm that this article content has no conflict of interest.

\section{ACKNOWLEDGEMENTS}

Declared none.

\section{REFERENCES}

[1] Hochberg MC, Silman AJ, Smolen JS, Weinblatt ME, Weisman MH. Hochberg textbook of rheumatology, $5^{\text {th }}$ ed. Mosby 2011; pp. 1351-9.

[2] Miyakis S, Lockshin, MD, Atsumi T, et al. International consensus statement on an update of the classification criteria for definite antiphosphoplipid syndrome (APS). J Thromb Haemost 2006; 4: 295-306.

[3] Liou HH, Wang CR, Chou HC, et al. Anticardiolipin antisera from lupus patients with seizures reduce a GABA receptor-mediated chloride current in snail neurons. Life Sci 1994; 54: 1119-25.

[4] Asherson RA, Hughes GRV. Antiphospholipid antibodies and chorea. J Rheumatol 1988; 15: 377-9.

[5] Cervera R, Piette JC, Font J, et al. Antiphospholipid syndrome: clinical and immunologic manifestations and patterns of disease expression in a cohort of 1,000 patients. Arthritis Rheumat 2002; 46: 1019-27.

[6] Bick RL, Baker WF. Antiphospholipid syndrome and thrombosis; Semin Thromb Hemost 1999; 25(3): 333-50.

[7] Baker WF Jr, Bick RL. The clinical spectrum of antiphospholipid syndrome. The Hematol Oncol Clin North Am 2008; 22(1): 33-52.

[8] Vlachoyiannopoulos PG, Kanellopoulos PG, Ioannidis JPA, Tektonidou MG, Mastorakou I, Moutsopoulos HM. Atherosclerosis in premenopausal women with antiphospholipid syndrome and systemic lupus erythematosus: a controlled study. Rheumatology 2003; 42: 645-51.

[9] Alves DJ, Ames PRJ, Donohue S, et al. Antibodies to high-density lipoprotein and $\beta_{2}$-glycoprotein I are inversely correlated with paraoxonase activity in systemic lupus erythematosus and primary antiphospholipid syndrome. Arthritis Rheumat 2002; 46: 2686-94.

[10] George J, Shoenfeld Y, Harats D. The involvement of beta 2glycoprotein-I in human and murine atherosclerosis. J Autoimmun 1999; 13: 57-60
[11] Ames PR, Margarita A, Delgado Alves J, Tommasino C, Iannaccone L, Brancaccio V. Anticardiolipin antibody titre and plasma homocysteine level independently predict intima media thickness of carotid arteries in subjects with idiopathic antiphospholipid antibodies. Lupus 2002; 11(4): 208-14.

[12] Perl L, Netzer A, Rechavia E, et al. Long-term outcome of patients with antiphospholipid syndrome who undergo percutaneous coronary intervention: Cardiology 2012; 122(2): 76-82.

[13] Long BR, Leya F. The role of antiphospholipid syndrome in cardiovascular disease. Hematol Oncol Clin North Am 2008; 22(1): 79-94.

[14] Nesher G, Ilany J, Rosenmann D, Abraham AS. Valvular dysfunction in antiphospholipid syndrome: prevalence, clinical features, and treatment. Semin Arthritis Rheum 1997; 27(1): 27-35.

[15] Garcia-Torres R, Amigo MC, de la Rosa A, Moro'n A, Reyes PA. Valvular heart disease in primary antiphospholipid syndrome (PAPS): clinical and morphological findings. Lupus 1996; 5(1): 5661.

[16] Ford HJ, Roubey RA. Pulmonary manifestations of the antiphospholipid antibody syndrome. Clin Chest Med 2010; 31: 537-45.

[17] Espinosa G, Cervera R, Font J, Asherson RA. The lung in the antiphospholipid syndrome. Ann Rheum Dis 2002; 61(3): 195-98.

[18] Cartin-Ceba R, Peikert T, Ashrani A, et al. Primary antiphospholipid syndrome-associated diffuse alveolar hemorrhage. Arthritis Care Res (Hoboken) 2014; 66(2): 301-10.

[19] Francès C, Niang S, Laffitte E, Pelletier Fl, Costedoat N, Piette JC. Dermatologic manifestations of the antiphospholipid syndrome: Two hundred consecutive cases. Arthritis Rheumat 2005; 52: 178593.

[20] Frances C. Dermatological manifestations of Hughes' antiphospholipid antibody syndrome. Lupus 2010; 19(9): 1071-7.

[21] Weenig RH, Davis MD, Dahl PR, Su WP. Skin ulcers misdiagnosed as pyoderma Gangrenosum. N Engl J Med 2002; 347(18): 1412-8.

[22] Espinosa G, Cervera R. Clinical management of antiphospholipid syndrome-related thrombosis. Open Autoimmunity J 2010; 2: 6775.

[23] Levine SR, Deegan MJ, Futrell N, Welch KM. Cerebrovascular and neurologic disease associated with antiphospholipid antibodies: 48 cases. Neurology 1990; 40: 1181-9.

[24] Cuadrado MJ, Khamashta MA, D'Cruz D, Hughes GR. Migraine in Hughes syndrome- - heparin as a therapeutic trial? QJM Int J Med 2001; 94: 114-5.

[25] Sanna G, Bertolaccini ML, Cuadrado MJ, Khamashta MA, Hughes GRV. Central nervous system involvement in the antiphospholipid (Hughes) syndrome. Rheumatology (Oxford) 2003; 42(2): 200-13.

[26] Beh SC, Greenberg BM, Frohman T, Frohman EM. Transverse Myelitis. Neurol Clin 2013; 13(1): 79-138.

[27] Cuadrado MJ, Khamashta MA, Ballesteros A, Godfrey T, Simon MJ, Hughes GRV. Can neurologic manifestations of Hughes (antiphospholipid) syndrome be distinguished from multiple sclerosis? Analysis of 27 patients and review of the literature. Medicine (Baltimore) 2000; 79(1): 57-68.

[28] Toubi E, Khamashta MA, Panarra A, Hughes GR. Association of antiphospholipid antibodies with central nervous system disease in systemic lupus erythematosus. Am J Med 1995; 99(4): 397-401.

[29] Vasoo S, Sangle S, Zain M, D'Cruz D, Hughes G. Orthopedic manifestations of the antiphospholipid (Hughes) syndrome. Lupus 2005; 14(5), 339-345.

[30] Uthman I, Salti I, Khamashta MA. Endocrinologic manifestations of the antiphospholipid syndrome. Lupus 2006; 15(8), 485-9.

[31] Andre M, Delevaux I, Amoura Z, et al. Ovarian vein thrombosis in the antiphospholipid syndrome. Arthritis Rheum 2004; 50(1): 18386.

[32] Leder AN, Flansbaum B, Zandman-Goddard G, Asherson R, Shoenfeld Y. Antiphospholipid syndrome induced by HIV. Lupus 2001; 10(5): 370-4.

[33] Pandolfi C, Gianini A, Fregoni V, Nalli G, Faggi L. Hypopituitarism and antiphospholipid syndrome. Minerva Endocrinol 1997; 22(4): 103-105. 
[34] Andre M, Aumaitre O, Piette JC, Thieblot P. Hypopituitarism in a woman with a severe primary antiphospholipid syndrome. Ann Rheum Dis 1998; 57(4): 257-58.

[35] Baker WF Jr, Bick RL. The clinical spectrum of antiphospholipid syndrome. Hematol Oncol Clin North Am 2008; 22(1):33-52.

[36] Cervera R. Update on the diagnosis, treatment, and prognosis of the catastrophic antiphospholipid syndrome. Curr Rheumatol Rep 2010; 12(1):70-76.

[37] Homepage on the internet. Available from: www.med.ub.es/ MIMM UN/FORUM/CAPS.HTM. Updated: 22 January 2014.

[38] Asherson RA, Cervera R, de Groot PG, et al. CAPS: International consensus on classification criteria and treatment guidelines. Lupus 2003; 12(7):530-4.PMID: 12892393

[39] Arnaud L, Mathian A, Ruffatti A, et al. Efficacy of aspirin for the primary prevention of thrombosis in patients with antiphospholipid antibodies: an international and collaborative meta-analysis. Autoimmune Rev 2014; 13(3) 281-91.

[40] Erkan D, Harrison MJ, Levy R, et al. Aspirin for primary thrombosis prevention in the Antiphospholipid syndrome: a randomized double blind, placebo controlled trial in asymptomatic antiphospholipid antibody positive individuals. Arthritis Rheum 2007; 56(7): 238291.

[41] Nalli C, Andreoli L, Casu C, Tincani A. Management of recurrent thrombosis in antiphospholipid syndrome. Curr Rheumatol Rep 2014; 16(3): 405.

[42] Mehta TP, Smythe MA, Mattson JC. Strategies for managing heparin therapy in patients with antiphospholipid antibody syndrome. Pharmacotherapy 2011; 31(12): 1221-31.

[43] Ruis-Irastorza G, Crowther M, Branch W, Khamashta MA. Antiphospholipid syndrome. Lancet 2010; 376(9751): 1498-509.

[44] Erkan D, Aguiar CL, Andrade D, et al. $14^{\text {th }}$ international congress on antiphospholipid antibodies task force report on antiphospholipid syndrome treatment trends. Autoimmun Rev 2014; 13(6): 685-96.

[45] Sciascia S, Khamashta MA, D'Cruz DP. Targeted therapy in antiphospholipid syndrome. Curr opin Rheumatol 2014; 26(3): $269-75$.

[46] Connoly SJ, Ezekowitz MD, Yusuf S, et al. Dabigatran versus Warfarin in patients with Atrial Fibrillation. N Engl J Med 2009; 361(12): 1139-51.

[47] Patel MR, Mahaffey KW, Garg J, et al. Rivaroxaban versus warfarin in nonvalvular atrial fibrillation. N Engl J Med 2011; 365: 1557-9.

[48] Arachchillage DJ, Cohen H. Use of newer anticoagulants in antiphospholipid syndrome, Curr Rheum Rep 2013; 15: 1-9.

[49] Tektonidou MG, Laskari K, Panagiotakos DB, Moutsopoulos HM. Risk factors for thrombosis and primary thrombosis prevention in patients with SLE with or without antiphospholipid syndrome. Arthritis Rheum 2009; 61: 29-36.

[50] Kaiser R, Cleveland CM, Criswell LA. Risk and protective factors for thrombosis in SLE: results from a large multi-ethnic cogort. Ann Rheum Dis 2009; 68: 238-41.

[51] Girardi G. Pravastatin prevents miscarriages in antiphospholipid treated mice. J Reprod Immol 2009; 82: 126-31.

[52] Lopez-Pedrera C, Ruiz-Limon P, Aguirre MA, et al. Global effects of Fluvastatin on the prothrombotic status patients with antiphospholipid syndrome. Ann Rheum Dis 2011; 70: 675-82.

[53] Lopez-Pedrera C, Ruiz-Limon C, Aguirre MA, Rodriguez-Ariza A, Caudrado MJ. Potential use of statins in treatment of APS. Curr Rheumatol Rep 2012; 14:87-94.

[54] Lockshin MD, Pierangeli SS. Statins for the treatment of obstretic complications in antiphospholipid syndrome? J Reprod Immol 2010; 84: 206

[55] Erre GL, Pardini S, Faedda R, Passiue G. Effect of Rituximab on clinical and laboratory features of antiphospholipid syndrome: a case report and a review of literature. Lupus 2008;17: 50-5.

[56] Bakshi J, Stevens R. Rituximab therapy for recurrent thromboembolic disease in antiphospholipid syndrome. Lupus 2013; 22: 865-7.

[57] Berman H, Rodriguez- Pinto, Cervera R, et al. Rituximab use in the catastrophic antiphospholipid syndrome: descriptive analysis of the CAPS registry patients receiving rituximab.Autoimm Rev 2013; 12: $1085-90$.
[58] Erkan D, Vega J, Ramon G, Kozora E, Lockshin MD. A pilot open label phse II trial of Rituximab for non-criteria manifestations of antiphospholipid syndrome. Arthritis Rheum 2013; 65: 464-71.

[59] Espinosa G, Berman H, Cervera R. Managemet of refractory cases of catastrophic antiphospholipid syndrome. Autoimmun Rev 2011; 10: 664-8.

[60] Lonze BE, Singer AL, Montgomery RA. Eculizumab and renal transplant in a patient with CAPS. N Engl J Med 2010; 362(18): 1744-5.

[61] Agmon-Levin N, Blank M, Zandman-Goddard G, et al. Vitamin D: an instrumental factor in the anti-phospholipid syndrome by inhibition of tissue factor expression. Ann Rheum Dis 2011;70: 145-50.

[62] Sciascia S, Giachino O, Roccatello D. Prevention of thrombosis relapse in APS patients refractory to conventional therapy using intravenous immunoglobulin. Clin Exp Rheumatol 2012; 30: 40913.

[63] Tenti S, Guidelli GM, Bellisai F, Galaeazzi M, Fioranvanti A Long term treatment of antiphospholipid syndrome with intravenous immunoglobulin in addition to conventional therapy. Clin Exp Rheumatol 2013; 31(6): 877-82.

[64] Cervera R, CAPS Registry Project Group. Catastrophic antiphospholipid syndrome (CAPS): update from the 'CAPS Registry'. Lupus 2010;19(4): 412-8.

[65] Bucciarelli S, Espinosa G, Cervera R, et al. Mortality in the Catastrophic antiphospholipid syndrome: causes of death and prognostic factors in a series of 250 patients. Arthritis Rheum 2006; 54(8): 2568-76.

[66] Cuadrado MJ, Bertolaccini ML, Seed P, et al. Primary prevention of thrombosis in antiphospholipid antibodies positive patients: a prospective, multicenter, randomised, open trial comparing low dose aspirin with low dose aspirin plus low intensity oral anticoagulation. Arthritis Rheum 2009; 60: 1285.

[67] Danowski A, Rego J, Kakehasi AM, et al. Guidelines for treatment of antiphospholipid syndrome. Rev Bras Reumatol 2013; 53: 18492.

[68] Johnson R, Charnley J. Hydroxychloroquine in prophylaxis of PE following hip arthroplasty. Clin Orthop Relat Res 1979; (144): 1747.

[69] Ruiz-Irastorza G, Ramos-Casals, Brito- Zeron, Khamashta MA Clinical efficacy and side effects of antimalarials in systemic lupus erythematosus- a systematic review. Ann Rheum Dis 2010; 69(1): 20-8.

[70] Erkan D, Yazici Y, Peterson MG, Sammaritano L, Lockshin MD. A cross sectional study of clinical thrombotic risk factots and preventive treatments in antiphospholipid syndrome. Rheumatology (Oxford) 2002; 41(8): 924-9.

[71] Ruiz-Irastorza G, Hunt BJ, Khamashta MA. A systematic review of secondary thromboprophylaxis in patients with antiphospholipid antibodies. Arthritis Rheum 2007; 57: 1487-95.

[72] Sinthiya P, Khamashta MA. Duration of anticoagulation treatment for thrombosis in APS: is it ever safe to stop. Curr Rheumatol Rep 2013; 15(4): 318.

[73] Lockshin M, Tenedios F, Petri M, et al. Cardiac disease in the antiphospholipid syndrome: recommendations for treatment. Committee consensus report. Lupus 2003; 12: 518-23.

[74] Cervera R, Tektonidou MG, Espinosa G, et al. Task force on Catastrophic antiphospholipid syndrome and non- criteria APS manifestations-catastrophic APS, APS nephropathy and heart valve lesions. Lupus 2011; 20, 165-73.

[75] Erdozain JG, Ruiz-Irastorza, Segura MI, et al. Cardiac valve replacement in patients with antiphospholipid syndrome. Arthritis Care Res 2012; 64: 1256-60.

[76] Weinstein S, Piette W. Cutaneous manifestations of antiphopholipid antibody syndrome. Hematol Oncol Clin N Am 2008; 22: 67-77.

[77] Rodrigues C, Carvalho JF, Shoenfeld Y. Neurologic manifestations of antiphospholipid syndrome. Eur J Clin Invest 2010; 40: 350-9.

[78] Levin SR, Brey RL, Tilley BC, et al. Antiphospholipid antibodies and subsequent thrombo-occlusive events in patients with ischemic stroke. JAMA 2004; 291(5): 576-84 . 
[79] Sanna G, Bertolaccini ML, Cuadrado MJ, Khamashta MA, Hughes GRV. Central nervous system involvement in the antiphospholipid (Hughes) syndrome. Rheumatology (Oxford) 2003; 42: 200-13.

[80] Petri M. Pathogenesis and treatment of antiphospholipid antibody syndrome. Med Clin North Am 1997; 81(1): 151-77.

[81] Uthman I, Godeau B, Taher A, Khamashta MA. The hematologic manifestations of the antiphospholipid antibody syndrome. Blood Rev 2008; 22: 187-94.

[82] Espinosa G, Bucciarelli S, Cervera R, et al. Thrombotic microangiopathic hemolytic anemia and antiphospholipid antibodies. Ann Rheum Dis 2004; 63: 730-6.
[83] Espinosa G, Cervera R, Font J, Asherson RA. Adrenal involvement in the antiphospholipid antibody syndrome. Lupus 2003; 12: 56972 .

[84] Erkan D, Cervera R, Asherson R. Catastrophic Antiphospholipid Syndrome. Where do we stand? Arthritis Rheum 2003; 48: 3320-7.

[85] Bazan CE, Lopez DC, Lozano MP, Cervera R, Espinosa G. Discontinuation of anticoagulation or anti aggregation treatment may be safe in patients with primary antiphospholipid syndrome when antiphospholipid antibodies became persistently negative. Immunol Res 2013; 56: 358-61.

(c) Katikaneni et al.; Licensee Bentham Open.

This is an open access article licensed under the terms of the Creative Commons Attribution Non-Commercial License (http://creativecommons.org/licenses/by-nc/3.0/) which permits unrestricted, non-commercial use, distribution and reproduction in any medium, provided the work is properly cited. 